\section{(1)}

CrossMark

\title{
Tuberculosis re-treatment after exclusion of rifampicin resistance
}

\author{
To the Editor:
}

We agree with FALzon et al. [1] that treatment of isoniazid-resistant tuberculosis (TB) is an important issue. The first-line re-treatment regimen for previously treated TB patients, which was known as the category II regimen, comprised 8 months of isoniazid, rifampicin and ethambutol supplemented by streptomycin for the initial 2 months, and pyrazinamide for the initial 3 months (2SHRZE/HRZE/ 5HRE). It was introduced in the collaborative programme of the International Union Against Tuberculosis and Lung Disease in which the first-line initial regimen for new TB patients was an 8-month regimen comprising 2 months of isoniazid, rifampicin, pyrazinamide and streptomycin, followed by 6 months of isoniazid and thioacetazone (2HRZS/6HT) [2]. At that time, the prevalence of multidrug-resistant (MDR)-TB among previously treated TB cases was relatively low and the challenge was mainly isoniazid resistance [3]. The category II regimen was designed to overcome isoniazid resistance and to reduce the risk of acquired resistance to rifampicin. It performed fairly well in settings where resistance to isoniazid predominated among previously treated TB patients [4]. However, with the wide use of a 6-month regimen including rifampicin throughout the whole treatment course (2HRZE/ $4 \mathrm{HR}$ ) for new TB patients (category I regimen), the prevalence of rifampicin resistance among previously treated TB patients increased substantially [5], rendering the category II regimen ineffective and problematic.

In 2017, World Health Organization (WHO) recommended that in "patients who require TB retreatment, the category II regimen should no longer be prescribed and drug-susceptibility testing should be conducted to inform the choice of treatment regimen" [6]. It is indeed crucial to perform rapid drug susceptibility testing to detect rifampicin resistance. After the exclusion of rifampicin resistance, the issue of isoniazid resistance remains and the challenge of patients with isoniazid resistance without rifampicin resistance cannot be neglected [7].

While recommending not using the category II regimen, the Guideline Development Group expressed concern regarding treatment of patients with isoniazid-resistant TB and advised that "Providers must be vigilant about the possibility of isoniazid resistance and, if it is suspected, they must test for isoniazid susceptibility and treat accordingly" [6]. Unfortunately, the most widely available rapid drug-susceptibility testing (DST) is Xpert MTB/RIF (Cepheid, Sunnyvale, CA, USA), which detects resistance to rifampicin but not isoniazid. Line probe assays can detect isoniazid resistance but are not easily accessible. A small fraction of re-treatment TB patients may have conventional DST but it is not uncommon to have a long turnaround time in high TB burden countries. A newly developed cartridge using the GeneXpert platform for the rapid molecular detection of resistance to fluoroquinolones, aminoglycosides and isoniazid has very high sensitivity and specificity in the detection of isoniazid resistance but is not yet widely available [8].

This raises an important question: what regimen should be used in re-treatment TB after rifampicin resistance has been excluded by the Xpert test? The WHO guidelines indicated that "on the basis of the drug susceptibility profile, a standard first-line treatment regimen $(2 \mathrm{HRZE} / 4 \mathrm{HR})$ can be repeated if no resistance is documented" [6]; however, it did not provide clear guidance on regimens to be used when the DST of isoniazid is lacking. It is highly likely that the category I regimen will be used in TB retreatment (personal communication from the national TB programme of the Philippines and Viet Nam). The problem is that the category I regimen may not necessarily be better than the category II regimen in $\mathrm{TB}$ re-treatment, especially in patients with isoniazid-resistant TB.

@ERSpublications

Once rifampicin resistance is excluded, it might be wiser to use the category II regimen in TB retreatment http://ow.ly/KLJE30hDX0I

Cite this article as: Chiang C-Y, Trébucq A. Tuberculosis re-treatment after exclusion of rifampicin resistance. Eur Respir J 2018; 51: 1702282 [https://doi.org/10.1183/13993003.02282-2017]. 
TABLE 1 Failure, relapse and acquired drug resistance in new tuberculosis patients with isoniazid resistance without rifampicin resistance treated with the World Health Organization (WHO) new ${ }^{\#}$ regimen and in previously treated tuberculosis patients treated with the WHO re-treatment ${ }^{\text {? }}$ regimen

\begin{tabular}{lcc} 
& \multicolumn{2}{c}{ Pooled event rate+ (95\% CI) } \\
\cline { 2 - 3 } & New & Re-treatment \\
\hline Failure & $11 \%(6-17 \%)$ & $6 \%(2-10 \%)$ \\
Relapse & $10 \%(5-15 \%)$ & $5 \%(2-8 \%)$ \\
Acquired resistance & $8 \%(3-13 \%)$ & $3 \%(0-6 \%)$ \\
$\quad$ Acquired resistance that is multidrug resistance & $98 \%(92-99 \%)$ & $71 \%(29-96 \%)$
\end{tabular}

For treatment failure, pooled event rates did not differ significantly between those given the new and those given the re-treatment regimen. For relapse, pooled event rates differed significantly between those given the new and those given the re-treatment regimen $(p=0.02)$. For acquired drug resistance, pooled event rates differed significantly between those given the new and those given the re-treatment regimen $(p=0.02)$. \#: WHO standard 6-month regimen for new tuberculosis patients (2HRZE/4HR); ": WHO standard 8-month regimen for previously treated TB patients (2SHRZE/HRZE/5HRE); ${ }^{+}$: cumulative percentage associated with the outcome. Reproduced and modified from [9, tables 1 and 4] with permission from the publisher.

Meta-analysis shows that the pooled event rates of failure, relapse and acquired resistance in previously treated patients with isoniazid resistance who were treated with the category II regimen were all lower than those in new TB patients with isoniazid resistance who were treated with the category I regimen: $6 \%$ versus $11 \%$ for failure, $5 \%$ versus $10 \%$ for relapse and $3 \%$ versus $8 \%$ for acquired drug resistance (table 1) [9]. Furthermore, the proportion of acquired resistance that is MDR-TB was 71\% (95\% CI 29-96\%) in the former group and 98\% (95\% CI 92-99\%) in the latter group. This provided indirect evidence that by using effective drugs together with rifampicin for a longer period, the category II regimen may outperform the category I regimen in TB re-treatment, especially in protecting rifampicin, which is in line with the findings of another network meta-analysis [10]. This is not surprising because the category II regimen was initially designed to address isoniazid resistance in TB retreatment.

Using a 6-month category I regimen in TB re-treatment after rifampicin resistance being excluded may run a higher risk of acquired resistance to rifampicin than using the category II regimen in settings where isoniazid monoresistance is substantial and obtaining results of DST of isoniazid in a timely manner is not feasible. It might be wiser not to abandon the category II regimen prematurely before a better option is available in TB re-treatment.

Chen-Yuan Chiang ${ }^{1,2,3}$ and Arnaud Trébucq ${ }^{1}$

${ }^{1}$ International Union Against Tuberculosis and Lung Disease, Paris, France. ${ }^{2}$ Division of Pulmonary Medicine, Dept of Internal Medicine, Wan Fang Hospital, Taipei Medical University, Taipei, Taiwan. ${ }^{3}$ Division of Pulmonary Medicine, Dept of Internal Medicine, School of Medicine, College of Medicine, Taipei Medical University, Taipei, Taiwan.

Correspondence: Chen-Yuan Chiang, 111 Hsin-Long Road, Section 3, Taipei, 116, Taiwan.

E-mail: cychiang@theunion.org

Received: Nov 032017 | Accepted after revision: Dec 042017

Conflict of interest: None declared.

\section{References}

1 Falzon D, Schünemann HJ, Harausz E, et al. World Health Organization treatment guidelines for drug-resistant tuberculosis, 2016 update. Eur Respir J 2017; 49: 1602308.

2 Enarson DA. Principles of IUATLD Collaborative Tuberculosis Programmes. Bull Int Union Tuberc Lung Dis 1991; 66: 195-200.

3 Trébucq A, Anagonou S, Gninafon M, et al. Prevalence of primary and acquired resistance of Mycobacterium tuberculosis to antituberculosis drugs in Benin after 12 years of short-course chemotherapy. Int J Tuberc Lung Dis 1999; 3: 466-470.

4 Gninafon M, Tawo L, Kassa L, et al. Outcome of tuberculosis retreatment in routine conditions in Cotonou, Benin. Int J Tuberc Lung Dis 2004; 8: 1242-1247.

5 Zignol M, van Gemert W, Falzon D, et al. Surveillance of anti-tuberculosis drug resistance in the world: an updated analysis, 2007-2010. Bull World Health Organ 2012; 90: 111-119.

6 World Health Organization. Guidelines for treatment of drug-susceptible tuberculosis and patient care. 2017 update. World Health Organization Document 2017: WHO/HTM/TB/2017.05. Geneva, WHO, 2017. 
7 Stagg HR, Lipman MC, McHugh TD, et al. Isoniazid-resistant tuberculosis: a cause of concern? Int J Tuberc Lung Dis 2017; 21: 129-139.

8 Xie YL, Chakravorty S, Armstrong DT, et al. Evaluation of a rapid molecular drug-susceptibility test for tuberculosis. N Engl J Med 2017; 377: 1043-1054.

9 Gegia M, Winters N, Benedetti A, et al. Treatment of isoniazid-resistant tuberculosis with first-line drugs: a systematic review and meta-analysis. Lancet Infect Dis 2017; 17: 223-234.

10 Stagg HR, Harris RJ, Hatherell HA, et al. What are the most efficacious treatment regimens for isoniazid-resistant tuberculosis? A systematic review and network meta-analysis. Thorax 2016; 71: 940-949.

Copyright @eERS 2018 